\title{
Improvement of IT Industry Training Management System Based on Big Data Background
}

\author{
Hui Wang ${ }^{\mathrm{a},{ }^{*}, \text { Meina Chen }}$ \\ Department of Information Management, Dalian Neusoft University of Information, China. \\ *, a wanghui_xg@neusoft.edu.cn
}

Keywords: B/S; training management system; software engineering.

\begin{abstract}
In order to improve the management level and efficiency, and effectively reduce the labour intensity of managers, user's functional requirements for the training management system were analysed at the beginning and then the development method of the entire system was determined. The system architecture based on Web technology and B/S was used in order to complete the design and implementation of LAN government office automation system. The research results showed that the system could solve the information processing requirements in daily work. In a word, the system can be fully applied to the municipal office management, and effectively improve the management efficiency, reduce the labour intensity of managers, and achieve modern human management.
\end{abstract}

\section{Introduction}

The rapid development of information technology is triggering a profound change in production and life style, which has greatly promoted economic and social development. It also puts forward new requirements for the working methods of government administration. Information and efficiency are the features of this era. The maximum utilization of human resources and environmental resources is the fundamental guarantee for the competition and development of enterprises [1]. In the era of the current explosion of knowledge, the pace of knowledge renewal is accelerating. Training, as an important means of knowledge renewal, has been increasingly valued by companies and society [2]. The Labour and Social Security Bureau of each city manages a variety of training schools, playing an important part. With the continuous expansion of training business and the refinement of training processes, the requirements for training management are constantly improving, and objectively, the training management must be more scientific, standardized and efficient, so as to meet the growing demand for training services at present. [3].

Previous management method was mainly manual management, and all kinds of data had to be reported by all of the training schools. The statistics were cumbersome and the accuracy was not high. Thus, the work efficiency was very low. The training management system came into being under this demand. The completion and application of the system will provide an effective information platform for training management [4]. It can strictly control the information input of trainees and eliminate the behaviours of non-reporting and over-reporting of the trainees, which can improve the management level of the Municipal Labour and Social Security Bureau.

\section{Literature Review}

In the early 1980s, information technology gained a rapid development, and management information systems began to be widely used in various enterprises, assisting enterprises in rapid data processing, scientific management, production regulation and control, etc. [5]. Since 1990, the information management system has gradually developed into a new type of enterprise resource system, which is now widely known as ERP. It regards the company's internal procurement, production, sales as well as customers as a closely related supply chain, and integrates capital flow, material flow and information flow in an all-round way. It pursues operational efficiency and rational utilization of resources and improves the competitiveness of enterprises. At present, it is very common for enterprises in foreign countries to have ERP and advanced management experience. 
The development of information management system in China can be traced back to the mid-1980s when many large and medium-sized enterprises began to introduce personnel management systems and payroll management systems [6]. After 1990, Internet technology in China began to popularize rapidly, and various enterprises have set up network information management system in succession. In recent years, some large enterprises in China have just begun to introduce ERP.

\section{Methodology}

\subsection{Basic Concepts and Features of Big Data}

Big data is another hot topic in the Internet era after the emergence of cloud computing. According to CCW, an authoritative department, the direct commercial value of big data will reach 15.7 billion yuan in the coming year. Various companies, experts, and authorities have defined big data. However, there is no uniform standard yet. According to Gartner, a world-renowned consulting firm, big data is an information asset with the characteristics of large scale, diversity, and high-growth. Its structure is incompatible with the existing database processing systems. So, new parallel data processing platforms or technologies are needed to extract potentially valuable decisions and optimize information from big data. The Analyst team of the "PTZ" believes that big data is often used to describe the large amount of unstructured data and semi-structured data created by a company. It will take too much time and money to download these data to relational databases for analysis. Big data analysis is often associated with cloud computing because real-time, large-scale dataset analysis requires a framework like MapReduce to allocate jobs to dozens, hundreds, or even thousands of computers. Although the definitions are different, big data has typical $4 \mathrm{~V}$ characteristics which are internationally recognized. That is, Volume, Variety, Velocity, and Value.

\subsection{OWC Introduction}

Office Web Components is a set of COM controls designed to provide interactive spreadsheet modeling, data reporting, and data visualization capabilities for a wide range of control containers. The OWC library contains four main components: a spreadsheet component, a chart component, a PivotTable component, and a data source component.

The word "Office" in the name of "Office Web Components" indicates that these controls were developed by some of the programmers who developed Microsoft Excel and Access, and these controls are designed to look, feel, and behave like a streamlined version of their Microsoft Office brothers. These controls do not contain all the functions of Excel and Access. In other words, people will not download the complete Excel and Access dynamically in order to view a report in the browser. These controls do contain many common features, especially the ones which are necessary to interact with existing content. In addition, these controls can also read and write Excel 2000 HTML format files, allowing users to click a button to load the current data into Excel for more powerful analysis.

\section{Results and Discussions}

According to the results of the demand analysis, we have a deep understanding of the system's objectives and implementation techniques. The program's modules are designed in an overall way according to the software engineering method. According to the functions that the module needs to achieve, the database is designed to enable people to have a clearer understanding of the expected goals of the system.

User's detailed opinions and suggestions are solicited and then summarized before the design of the system in order to ensure that the system has the features of perfect function, easy operation, easy maintenance, safety and reliability.

\section{1 Function Module Design of Training Management System}

According to the functions required by the user business processing process and demand analysis, the functional modules of the training management system are divided. The system has the following 
modules: student information management, student archive printing, student information statistics, chart display, user management, teacher management, curriculum management, performance management and system management.

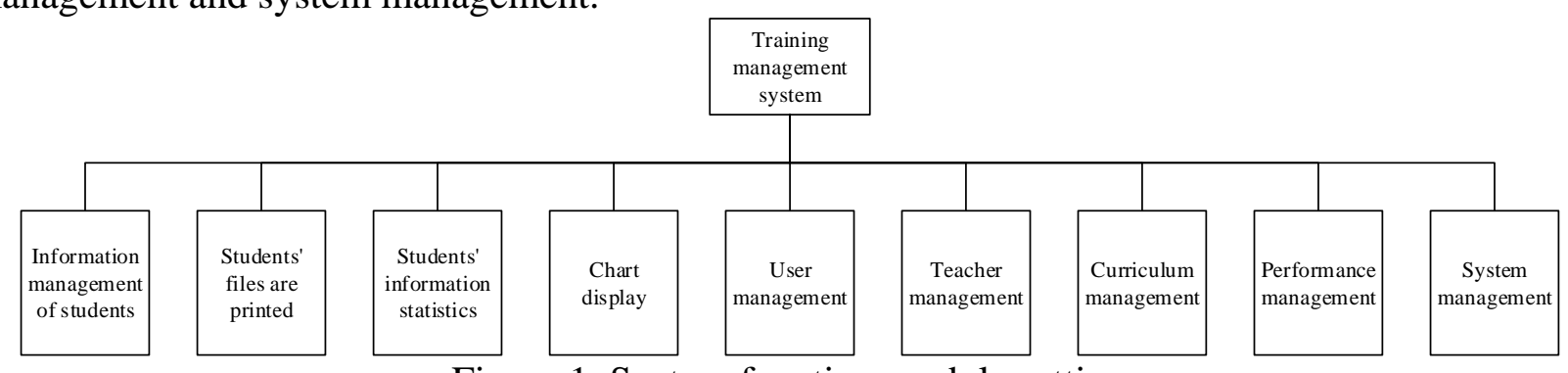

Figure 1. System function module setting

The core modules of the training management system include student information management, students' information statistics, students' file printing, graphic information display, user management and system management. The student information management module mainly realizes the function of entering, modifying, deleting and exporting of students' information. It can realize the input of individual student's information and the batch import of students' information through Excel. At the same time, students' information can also be exported to Excel. The students' information statistics mainly achieves the function of the statistics of various indicator structures of the overall students, such as the statistics of the sex ratio, training professional proportion statistics, the statistics of training categories, and so on. Students' file printing can print not only individual student's files but also batch students' files. The function of graph information display is to show the overall proportion of students in a more vivid way. It is clear, displaying charts and statistics of various indicators separately. User management can be used to add and delete users, and users can modify their own passwords. At the same time, it's necessary for the module to allocate different privilege functions for different users to ensure the security of the system and facilitate the management of the administrator. Teacher management mainly implements the addition, modification, and deletion of class teachers. The course management mainly realizes the function of adding, deleting and modifying training courses for trainees. Performance management mainly achieves the management of students' scores in all subjects. The teacher only has the ability to input grades but cannot make any changes, because grade changes can only be modified by the system administrator. System management is a function module possessed by the system administrator. It mainly realizes the maintenance of the basic data of the system, such as training school management, training professional management, training professional category management, etc., and can be used to add, modify and delete these basic data.

\section{2 Training Management System UML Use Case Diagram}

The definition of the user role is shown in Figure 2. The management of the system platform is shown in Figure 3.

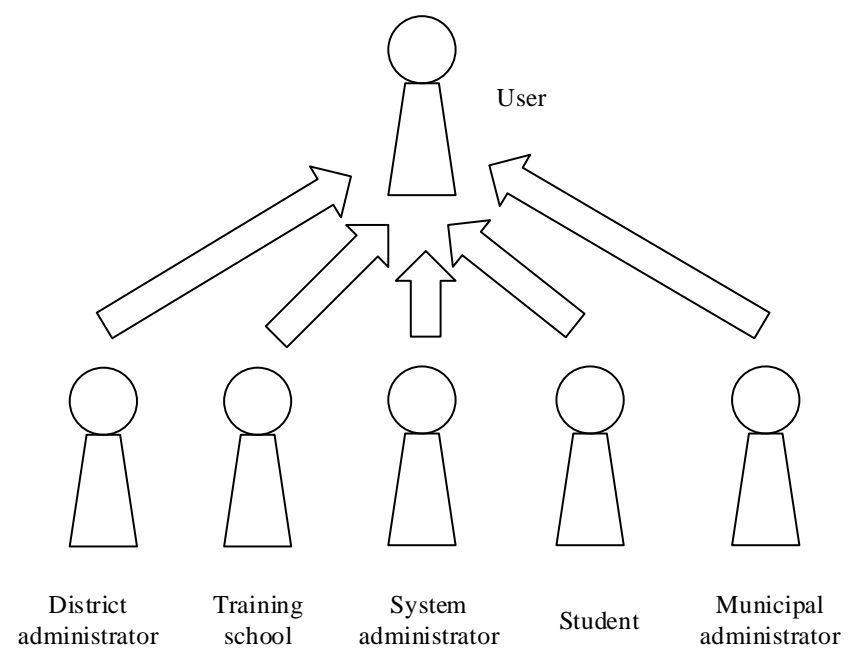

Figure 2. User roles 

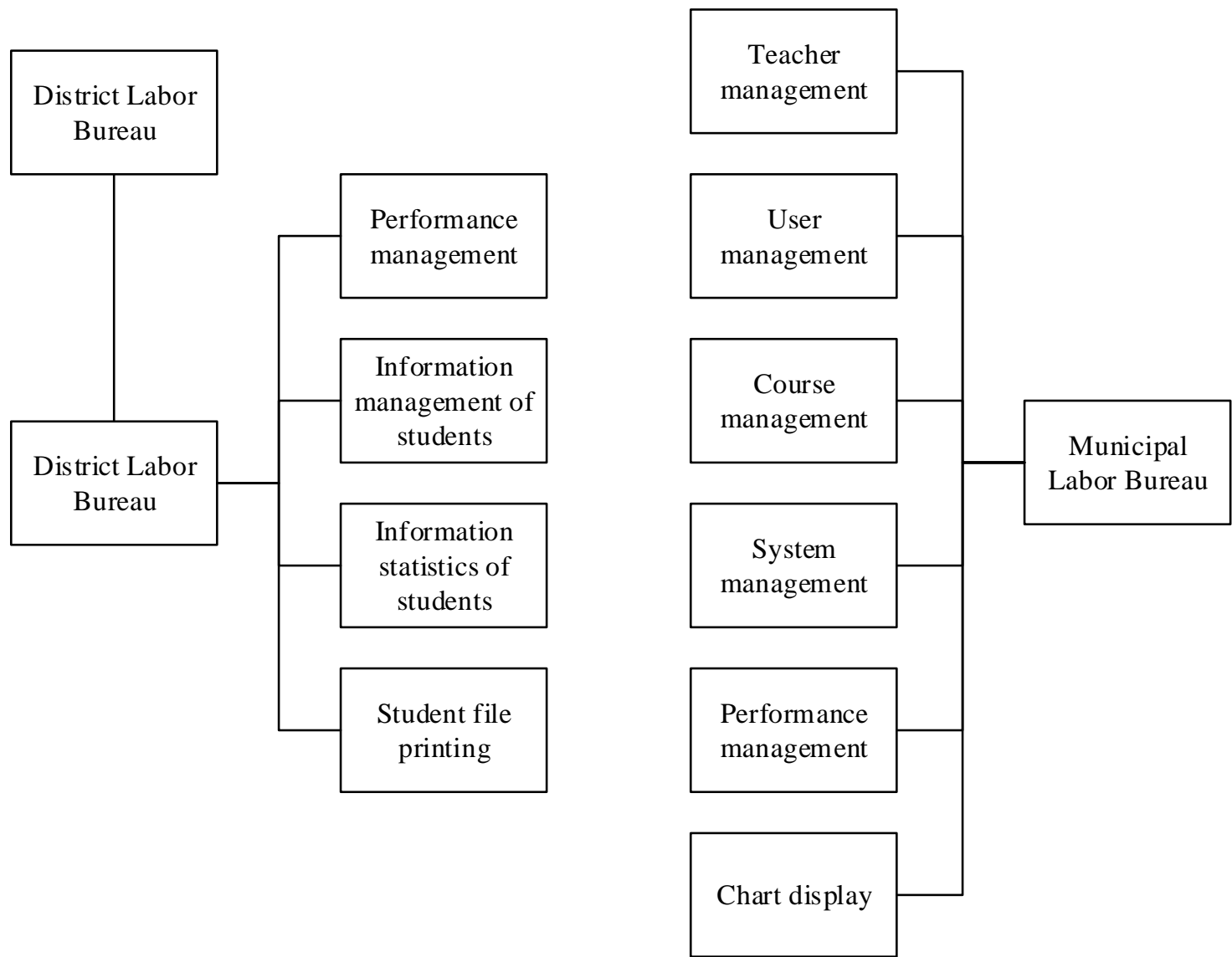

Figure 3. System platform management

\section{3 Database Design of Training Management System}

Database design refers to the establishment of a variety of data tables and the data storage mode in the table as well as the relationship setting between the tables in order to construct a database model with the best performance which can effectively store all the data and be beneficial to the system development. At the same time, it must also achieve the purpose of simplifying the development and meet the dual requirements of both users and developers.

The database plays a very important part in the management system. The design of the database structure will directly affect the efficiency of the application system and the effect of the implementation. A reasonable design of the database structure can improve the efficiency of data storage, ensure the integrity and consistency of the data, and be beneficial to the implementation of the program. The database of office automation systems is designed by using advanced data modeling software Power Designer.

As the core part of the management information system design, the database model is the organization, storage and description of the user business data from the logical layer to the physical layer, and the database model determines how the system is programmed.

According to the requirements of the training management system, the system takes the user as the core and the configuration table as the basis to conduct modeling based on documents, configuration files and related management information.

\section{4 Students' Statistics and Chart Display}

Students' statistics mainly implement statistical classification of 9 major data, including gender statistics, statistics of 4050 trainees, statistics of training course completion, professional statistics, training category statistics, student category statistics, training level statistics, rank statistics of professional skill identification, and statistics of the number of students in the partition.

In order to display the overall distribution of students more clearly, histograms, pie charts and polygon maps and so on were generated based on the data of the statistical analysis. In order to realize the drawing of various charts, Microsoft's OWC technology was adopted and Microsoft's Office 
Web Control was made use of in the chart generation. Various charts were dynamically generated based on system data.

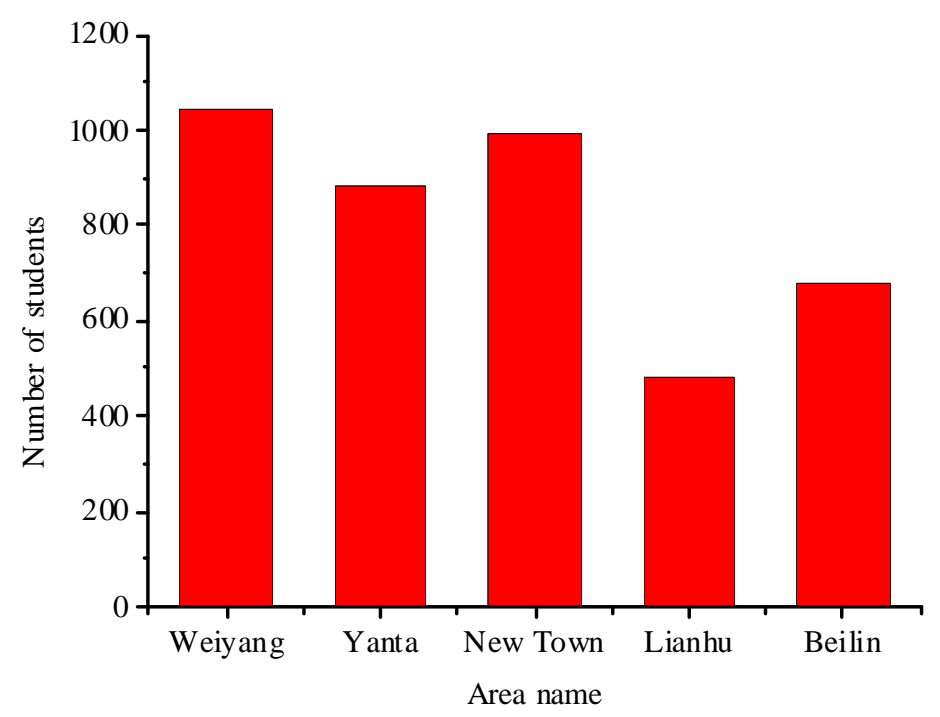

Figure 4. Student statistics in each area
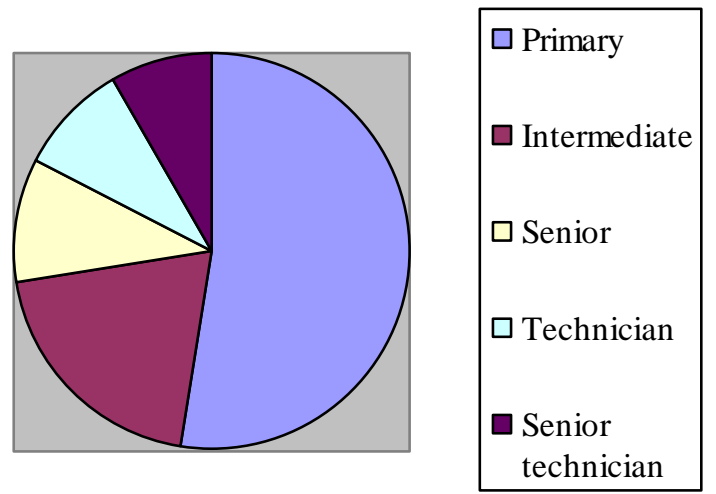

Figure 5. Professional skill identification level

\section{Conclusion}

This article analyzes and summarizes the functional requirements of the training management system in detail. The client accesses the WEB server and its linked back-end database through browser by taking WEB database as the center and HTTP as the transport protocol, that is, the system is designed by using the three-level BS system architecture. This paper describes the realization of the student information management function module in detail, and elaborates the management of student information input, modification and deletion. Finally, the chart display function is realized through the use of OWC technology, that is, the data indicators in the statistical analysis are graphically made clearer and clearer.

\section{References}

[1]. Yao F. Under the background of the big data age, the legislation of the Obama government's Information Sovereignty protection legislation brings us the enlightenment, trade and trade, 2014, 3(1): pp.29-32.

[2]. Zhu D, Zhang Y, Wang X, et al. Research on technology innovation management method for large data environment, Beijing Institute of Technology, 2013, 1 (3), pp.66-69. 
[3]. $\mathrm{Xu} \mathrm{H}$. On the transformation of the needs of competitive intelligence in the era of big data, electronic technology and software engineering, 2014, 32(1), pp.62-66.

[4]. Li X, Gong H. Summary of large data systems, summary of large data systems, 2015, 1(1), pp.60-73.

[5]. Xiong X. Empirical research on the impact of APP marketing on consumer buying behavior, Hubei University of Technology, 2014, 1(2), pp.56-62.

[6]. Wang X. Major data key technology, ZTE technology, 2013, 2(1), pp.221-222. 